\title{
The Minimum Power Broadcast problem in Wireless Networks: a Simulated Annealing approach
}

\author{
Roberto Montemanni, Luca Maria Gambardella \\ Istituto Dalle Molle di Studi sull'Intelligenza Artificiale (IDSIA) \\ Galleria 2, CH-6928 Manno-Lugano, Switzerland \\ Email addresses: \{roberto,luca\}@idsia.ch \\ Arindam K. Das \\ Department of Electrical Engineering, University of Washington \\ Box 352500, Seattle, WA 98195, U.S.A. \\ Email address: arindam@ee.washington.edu
}

\begin{abstract}
Broadcasting in wireless networks, unlike wired networks, inherently reaches several nodes with a single transmission. For omnidirectional wireless broadcast to a node, all nodes closer will also be reached. This property can be used to compute routing trees which minimize the sum of the transmitter powers.

In this paper we present a simulated annealing algorithm for the problem. Extensive experimental results are presented. They show that the algorithm we propose is capable of improving the results of state-of-the-art algorithms for most of the problems considered. The solutions provided by the simulated annealing algorithm can be improved by applying a very fast post-optimization procedure. This leads to the best known mean results for the problems considered.
\end{abstract}

Keywords-Wireless networks, minimum power broadcast, simulated annealing.

\section{INTRODUCTION}

Among the most crucial issues related to ad-hoc and sensor networks is that of operation in limited energy environments, since devices are usually equipped with battery with a limited lifetime.

Since radio signals have non-linear attenuation properties, it is very energy-consuming to transmit a signal far away. Another drawback of long-range transmissions is that they tend to produce widespread interference over the network, and for this reason they should be avoided.

The previous issues can be seen as correlated, and they can be handled together by taking advantage of the socalled wireless multicast advantage property (see, Wieselthier et al. [14]). This property is based on the observation that, in wireless networks, devices are usually equipped with omnidirectional antennae, and for this reason multiple nodes can be reached by a single transmission. If, for example, nodes $j$ and $k$ are closer to node $i$ than node $m$, then the signal originating in node $i$, and directed to node $m$, will be received also by nodes $j$ and $k$, since they are within the transmission range of a communication from

R.M. and L.M.G. were partially supported by the Future \& Emerging Technologies unit of the European Commission through project "BISON: Biology-Inspired techniques for Self Organization in dynamic Networks"(IST-2001-38923). node $i$ to node $m$.

For a given network with an identified source node, the $M P B$ (minimum power broadcast) problem is to assign transmission powers to the nodes in such a way that the network is connected and the total power consumption is minimized. The $M P B$ problem in wireless networks is shown to be NP-complete in Cagalj et al. [1], and this implies that polynomial time algorithms are unlikely to exist. Some mixed integer programming formulations for the problem are described in Das et al. [6].

Wieselthier et al. [14] first observed that the so called "node based" approach is more suitable for wireless environment than the previously adopted "link-based" algorithms. They developed the Broadcast Incremental Power $(B I P)$ algorithm, which is a simple sub-optimal heuristic for constructing minimum power broadcast trees in wireless networks. In this algorithm, new nodes are added to the tree on a minimum incremental cost basis, until all intended destination nodes are included. It was subsequently shown in Wan et al. [13] that the BIP algorithm has an approximation ratio between $13 / 3$ and 12 . Other techniques that have been suggested for solving this problem include an internal nodes based broadcasting produce by Stojmenovic et al. [12], an evolutionary approach by Mark et al. [9], a localized algorithm by Cartigny et al. [2], a swarm based procedure by Das et al. [4] (Ant Colony System, ACS, see also Gambardella and Dorigo [8]). This last algorithm was hybridized within a cluster-merge (CM) method presented in Das et al. [5]. Some heuristic approaches for improving solutions provided by other methods was presented in Das et al. [7]. Most of these heuristic techniques are described in detail in Das et al. [3].

The rest of the paper is organized as follows. In Section II, we outline the network model. The simulated annealing paradigm and its adaptation to the $M P B$ problem is presented in Section III. Computational results are presented in Section IV, while Section V contains conclusions. 


\section{NETWORK MODEL}

We assume a fixed $N$-node network with a specified source node which has to broadcast a message to all other nodes in the network. Any node can be used as a relay node to reach other nodes in the network. All nodes are assumed to have omnidirectional antennae, so that if node $i$ transmits to node $j$, all nodes closer to $i$ than $j$ will also receive the transmission.

The signal propagation we adopt, which is the most common one in the literature (see, for example, Wieselthier et al. [14], Stojmenovic et al. [12] and Das et al. [3]), works as follows. For a node $i$ of the network, the power required to reach another node $j$ is given by:

$$
p_{i j}=\left(d_{i j}\right)^{\kappa}
$$

where $d_{i j}$ is the Euclidean distance between nodes $i$ and $j$ and $2 \leq \kappa \leq 4$ is the channel loss exponent.

We assume that there is no constraint on maximum transmission power. However, the algorithm we discuss in this paper can be extended straightforwardly to the case where this assumption does not hold. If, for example, node $i$ cannot reach node $j$ even when it is transmitting at its maximum power (i.e. $d_{i j}^{\kappa}>$ maximum power of $i$ ), then $p_{i j}$ can be redefined as $+\infty$.

We consider a centralized implementation where construction of the routing tree is done at the source node, which has complete knowledge of the locations of all nodes in the network. Finally, we assume that power expenditures due to signal reception and processing are negligible compared to signal transmission and hence the cost of a routing tree is equal to the sum of transmitter powers corresponding to the set of edges chosen in the tree.

\section{Simulated AnNEAling Algorithm}

\section{A. General description}

Simulated annealing is a metaheuristic algorithm derived from thermodynamic principles. It has been applied originally to combinatorial optimization in Kirkpatrick et al. [10]. It can be used to find (near) minimum cost solutions ${ }^{1}$ of difficult problems characterized by vast search spaces, on which it is impossible to obtain the optimal solution by running exact algorithms.

The search proceeds with the cost function reducing most of the time, but it is allowed to increase sometimes to permit escape from local minima which are not global minima. The analogy with thermodynamics, and specifically with the way that liquids freeze and crystalize, or metals cool and anneal, is in the strategy adopted to accept or not accept cost-increasing solutions. At high temperatures, the molecules of a liquid move freely with respect to one another. If a liquid metal is cooled quickly (i.e. quenched), it does not reach a minimum energy state but a somewhat higher energy state corresponding, in the mathematical sense, to a suboptimal solution. On the other hand, if

\footnotetext{
${ }^{1}$ Here and in the following we suppose the methods to be applied to minimization problems. It is trivial to adapt the descriptions for the maximization case.
}

the liquid is cooled slowly, thermal mobility is restricted. The atoms are often able to line themselves up and form a pure crystal that is completely regular. The crystal is the state of minimum energy for the system, which corresponds to the optimal solution in a mathematical optimization problem. The algorithm is based on the connection of the physical concept of temperature with the mathematical concept of the probability of accepting a cost-increasing solution. The probability will be high initially and will decrease slowly, like the temperature in the annealing process which produces the regular crystal.

In the next section a mapping of the paradigm to the $M P B$ problem will be described.

\section{B. A simulated annealing algorithm for the MPB problem}

Each solution for the MPB problem is represented by the set of transmission powers assigned to the nodes of the network, while the fitness value of each solution (analogous to the energy of the system in the thermodynamic case) is represented by the sum of the transmission powers of all the nodes.

In our algorithm, only solutions which provide a connected broadcasting tree (i.e., feasible solutions) are considered, and the goal is to find a solution with minimum cost.

The starting solution for the SA algorithm is obtained from that provided by the BIP algorithm (see Wieselthier et al. [14]), a fast polynomial time constructive heuristic method. In order to provide the SA algorithm a richer search space, this solution is perturbed in such a way that it remains in the attraction-basin of the solution provided by BIP algorithm, but less deep inside the corresponding local minimum valley. This helps the algorithm to move to different local optima easily. Each node $i$ is considered and if it is not already transmitting at its maximum possible power (i.e. to reach the node farthest away from it, subject to its eventual maximum power constraint) then, with probability $p_{p}$, its power is increased in such a way that node $i$ can reach one more node. It is important to observe that with the given perturbation strategy, each initial solution is feasible since transmission powers can only be augmented (i.e. solution cost can only increase), starting from the values provided by BIP algorithm, which is feasible by definition.

Preliminary tests showed that the starting solution obtained as described above usually provides a better final solution than those obtained by computing (and eventually perturbing) the Minimum Spanning Tree (i.e. ignoring the wireless multicast advantage) using Prim's algorithm [11], or by running the Stochastic tree generation algorithm presented in Marks et al [9].

The SA algorithm then enters an iterative state, where the simulation of the annealing process is carried out. In this phase the broadcasting tree is repeatedly disconnected and repaired. The disconnect and repair strategies we adopt can be seen as a probabilistic version of the $r$-shrink tree-improvement algorithm described in Das et al. [7] and are explained below. 
In the remainder of this section, we will refer to the current solution (which is the initial solution during the first iteration) as $S_{O}$. At each iteration the following steps are carried out:

- A random node $i$ is selected among the ones transmitting in the current solution $S_{O}$.

- The power of node $i$ is decreased in such a way that it can reach one less node than in solution $S_{O}$. We will refer to the node which is not reached anymore by node $i$ as $j$. - If solution $S_{N}$ is still providing a feasible broadcasting tree - this happens if $i$ was using more power than necessary in solution $S_{O}$ - no operation is carried out on solution $S_{N}$. - If solution $S_{N}$ does not provide a feasible broadcasting tree anymore, the broadcasting subtree not containing node $j$ - we will refer to as $S u b T$ - is identified and one of its nodes, $k$, is selected. With probability $p_{r}$, node $k$ is selected at random among those with $p_{k j}<+\infty$, while with probability $\left(1-p_{r}\right)$ it is selected as the node of $S u b T$ which reconnects the broadcasting tree with the minimum increment in power.

- The new solution $S_{N}$ is accepted with probability given by:

$$
\min \left\{1, e^{-\frac{\operatorname{Cost}\left(S_{N}\right)-\operatorname{Cost}\left(S_{O}\right)}{t}}\right\}
$$

Note that not only improving solutions are accepted, but also solutions that do not improve the current one are sometimes accepted. Their acceptance probability is regulated by variable $t$, which is analogous to temperature in the real annealing process. Accepting non-improving solutions helps the algorithm to escape from local minima.

Temperature $t$, which initially assumes the value of parameter $t_{\text {init }}$, is decreased every time $C_{T}$ consecutive iterations are carried out without improving the best solution. This simulates the annealing process. The parameter $t$ is decreased as follows:

$$
t:=\alpha t
$$

where $0<\alpha<1$ is a user defined parameter regulating the annealing process.

In the beginning, the temperature $t$ is high and most of the new configurations are accepted. As the algorithm proceeds, $t$ is reduced until it reaches a value where nonimproving configurations are all rejected. When $t$ goes below a given threshold, $T_{t}$, the $\mathrm{SA}$ algorithm is stopped. The post-optimization algorithm Sweep (see Wieselthier et al. [14]), which has almost negligible computation times, is run after the SA algorithm terminates. A simplified pseudocode of the simulated annealing algorithm is presented in Figure 1.

\section{Computational Results}

The simulated annealing algorithm was tested on 25, 50, 75 and 100-node networks in a $5 \times 5$ grid. In each case, 50 networks were randomly generated and the tree powers were averaged to obtain the mean tree power.

Parameter $\kappa$ of equation (1) was chosen to be equal to 2 for all cases. Tests for the SA algorithm were carried out on a computer equipped with an Intel Celeron $1.3 \mathrm{GHz}$
$S_{O}:=\operatorname{BIP}()$

For $i:=1$ to $N$;

If $\left(\operatorname{rand}(0,1)<p_{p}\right)$

increase the power of $i$ in solution $S_{O}$;

Best $S:=S_{O}$;

$t:=t_{\text {init }}$

$C:=0$

While $\left(t<T_{t}\right)$

$\operatorname{If}\left(C>C_{T}\right)$

$t:=\alpha t$

$S_{N}:=S_{O}$

$C:=C+1$;

$i:=$ random transmitting node of $S_{N}$;

decrease the power of $i$ in $S_{N}$

(node $j$ is not reached anymore by $i$ );

If ( $S_{N}$ is a feasible solution)

Else $S_{O}:=S_{N}$

$S u b T:=$ subtree of $S_{N}$ not containing $j ;$

If $\left(\operatorname{rand}(0,1)<p_{r}\right)$ $k:=$ random node in $S u b T$;

Else

$k:=$ node in $S u b T$ which reconnects solution $S_{N}$ with the minimum increase in power;

$\operatorname{If}\left(\operatorname{rand}(0,1)<e^{-\frac{\operatorname{Cost}\left(S_{N}\right)-\operatorname{Cost}\left(S_{O}\right)}{t}}\right)$ $S_{O}:=S_{N}$

If $\left(\operatorname{Cost}\left(S_{N}\right)<\operatorname{Cost}(\operatorname{Best} S)\right)$

$\operatorname{Best} S:=S_{N}$

$C:=0$

Return BestS;

Fig. 1. Simulated annealing algorithm for the $M P B$ problem.

processor and $256 \mathrm{MB}$ of memory. The parameter settings adopted for the simulations are summarized in Table I.

It is important to observe that these parameter values guarantee quick solving times (no more than a few seconds for the biggest problems). Tests not reported in this paper also suggest that the simulated annealing based algorithm is not very sensitive to the changes in parameter values, which are almost independent from the problem dimensions.

Computational results are presented in Table II. In the first column the different networks considered are listed. In the remaining columns the mean tree powers for different algorithms are presented. In particular BIP, BIP followed by the sweep algorithm (see Wieselthier et al. [14]), ACS (see Das et al. [4]) and CM (see Das et al. [5]) followed by the 1-shrink algorithm (see Das et al. [7]) are considered together with the SA algorithm, which is the one discussed in this paper. The last column contains the results obtained by SA followed by the sweep algorithm. Percentage improvements in the mean tree powers over the BIP solutions are shown in Table III.

From Table II and Table III it can be seen that the 
TABLE I

Parameter Setting FOR the Simulated AnNEAling Algorithm.

\begin{tabular}{ccc}
\hline Parameter & Meaning & Value \\
\hline$p_{p}$ & Perturbation probability (initial solution) & 0.3 \\
$p_{r}$ & Random selection probability for reconnection & 0.2 \\
$t_{i n i t}$ & Initial temperature & 0.2 \\
$C_{T}$ & Iteration interval for temperature update & 30000 \\
$\alpha$ & Annealing parameter & 0.9 \\
$T_{t}$ & Stopping criterion (temperature threshold) & 0.1 \\
\hline
\end{tabular}

TABLE II

MEAN TREE POWERS OBTAINED BY DIFFERENT ALGORITHMS.

\begin{tabular}{ccccccc}
\hline$N$ & BIP & $\begin{array}{c}\text { BIP+ } \\
\text { sweep }\end{array}$ & ACS & $\begin{array}{c}\text { CM+ } \\
\text { 1-shrink }\end{array}$ & SA & $\begin{array}{c}\text { SA+ } \\
\text { sweep }\end{array}$ \\
\hline 25 & 12.46 & 12.14 & 10.21 & 10.23 & 9.98 & $\mathbf{9 . 9 5}$ \\
50 & 11.67 & 11.45 & 10.04 & 9.90 & 9.67 & $\mathbf{9 . 6 5}$ \\
75 & 11.63 & 11.37 & - & 9.88 & 9.84 & $\mathbf{9 . 7 4}$ \\
100 & 11.60 & 11.36 & - & 9.87 & 9.94 & $\mathbf{9 . 8 2}$ \\
\hline
\end{tabular}

TABLE III

Percentage improvements (\%) in mean tree power over BIP ALGORITHM.

\begin{tabular}{cccccc}
\hline$N$ & $\begin{array}{c}\text { BIP+ } \\
\text { sweep }\end{array}$ & ACS & $\begin{array}{c}\text { CM+ } \\
\text { 1-shrink }\end{array}$ & SA & $\begin{array}{c}\text { SA+ } \\
\text { sweep }\end{array}$ \\
\hline 25 & 2.57 & 18.06 & 17.90 & 19.90 & $\mathbf{2 0 . 1 4}$ \\
50 & 1.89 & 13.93 & 15.17 & 17.14 & $\mathbf{1 7 . 3 1}$ \\
75 & 2.24 & - & 15.05 & 15.39 & $\mathbf{1 6 . 2 5}$ \\
100 & 2.07 & - & 14.91 & 14.31 & $\mathbf{1 5 . 3 4}$ \\
\hline
\end{tabular}

SA algorithm is able to substantially improve the results achieved by the other algorithms for problems with up to 75 nodes. It works particularly well on small/medium size problems. On the other hand, it is comparable to the $\mathrm{CM}+$ 1-shrink algorithm for problems with 100 nodes. A small further improvement in the solutions provided by SA algorithm can be obtained by running the sweep algorithm, which - as observed in Section III-B - has negligible computation times, on them. This leads to the best mean results for problems with $|V|=100$.

\section{Conclusion}

In this paper, we have presented a new heuristic algorithm for solving the minimum power broadcast problem in wireless networks. It is based on the simulated annealing paradigm, which proves to be very suitable for the problem under consideration.

Experimental simulations show that the simulated annealing algorithm is able to provide high quality solutions, which are significantly better than those generated by the BIP algorithm. The solutions are also better than the best results reported thus far in the literature.

\section{REFERENCES}

[1] M. Cagalj, J.P. Hubaux, and C. Enz. Minimum-energy broadcast in all-wireless networks: NP-completeness and distribution issues. In Proceedings of the Mobicom 2002 Conference, Atlanta, GA, September 23-28, 2002.

[2] J. Cargigny, D. Simplot, and I. Stojmenović. Localized minimum-energy broadcasting in ad-hoc networks. In Proceedings of the IEEE Infocom 2003 Conference, San Francisco, CA, March 30 - April 3, 2003.

[3] A.K. Das. Minimum power broadcast and maximization of timeto-first-failure in broadcast wireless networks. PhD thesis, University of Washington, May 2003.

[4] A.K. Das, R.J. Marks, M. El-Sharkawi, P. Arabshahi, and A. Gray. The minimum power broadcast problem in wireless networks: an ant colony system approach. In Proceedings of the IEEE CAS Workshop on Wireless Communications and Networking, Pasadena, CA, September 5-6, 2002.

[5] A.K. Das, R.J. Marks, M. El-Sharkawi, P. Arabshahi, and A. Gray. A cluster-merge algorithm for solving the minimum power broadcast problem in large scale wireless networks. In Proceedings of the Milcom 2003 Conference, Boston, MA, October 13-16, 2003.

[6] A.K. Das, R.J. Marks, M. El-Sharkawi, P. Arabshahi, and A. Gray. Minimum power broadcast trees for wireless networks: integer programming formulations. In Proceedings of the IEEE Infocom 2003 Conference, San Francisco, CA, March 30 - April 3, 2003.

[7] A.K. Das, R.J. Marks, M. El-Sharkawi, P. Arabshahi, and A. Gray. $r$-shrink: A heuristic for improving minimum power broadcast trees in wireless networks. In Proceedings of the IEEE Globecom 2003 Conference, San Francisco, CA, December 1-5, 2003.

[8] L.M. Gambardella and M. Dorigo. Solving symmetric and asymmetric TSPs by ant colonies. In Proceedings of the IEEE ICEC 1996 Conference, Nagoya, Japan, May 20-22, pages 622-627, 1996.

[9] R.J. Marks II, A.K. Das, M. El-Sharkawi, P. Arabshahi, and A. Gray. Minimum power broadcast trees for wireless networks: optimizing using the viability lemma. In Proceedings of the IEEE International Symphosium on Circuits \& Systems, Scottsdale, AZ, May 26-29, 2002.

[10] S. Kirkpatrick, C.D. Gelatt, and M.D. Vecchi. Optimization by simulated annealing. Science, 220(4598):671-680, 1983.

[11] R.C. Prim. Shortest connection networks and some generalizations. Bell System Technical Journal, 36:1389-1401, 1957.

[12] I. Stojmenovic, M. Seddigh, and J. Zunic. Internal nodes based broadcasting in wireless networks. In Proceedings of the HICCS34 Conference, Island of Maui, HA, January 3-6, 2001.

[13] P.-J. Wan, G. Călinescu, X.-Y. Li, and O. Frieder. Minimumenergy broadcast routing in static ad hoc wireless networks. In Proceedings of the IEEE Infocom 2001 Conference, Anchorage, AL, April 22 - 26, 2001.

[14] J. Wieselthier, G. Nguyen, and A. Ephremides. On the construction of energy-efficient broadcast and multicast trees in wireless networks. In Proceedings of the IEEE Infocom 2000 Conference, Tel-Aviv, Israel, March 26 - 30, pages 585-594, 2000. 